\title{
ISI-reduced Modulation Over a Fading Multipath Channel ${ }^{\dagger}$
}

\author{
A. K. Khandani ${ }^{1}$ and P. Kabal ${ }^{1,2}$ \\ ${ }^{1}$ Dept. of Elec. Eng., McGill University, 3480 University, Montreal, Canada, H3A 2A7 \\ ${ }^{2}$ INRS-Telecommunications, 16 Place du Commerce, Verdun, Canada, H3E 1H6
}

\begin{abstract}
In this work, the idea of using the channel eigenvectors as the basis for a block based signaling scheme over a fading multipath channel is introduced. This basis minimizes the product of the average fading attenuations along different dimensions. The ISI from the preceding blocks (intra-block ISI) is modeled by an additive Gaussian noise. To reduce the effect of the intra-block ISI, a number of zeros are transmitted between successive blocks. The number of zeros is optimized to minimize the average probability of error. As the transmission of zeros reduces the bandwidth efficiency, this optimization procedure is more useful for lower bit rates. By applying Quadrature Amplitude Modulation (QAM) to each dimension, we obtain a set of two-dimensional subchannels with unequal fadings. A coherent M-PSK constellation is employed over each QAM subchannel. We propose two methods to distribute the rate and energy between the subchannels. In both methods, we impose the restriction that the average error probability for all the subchannels is the same. In the optimum method, the energy is distributed equally between the nonempty subchannels and the rate is distributed to obtain equal average error probabilities. In a second method, the rate is distributed equally and the energy is distributed to obtain equal average error probabilities. The second method allows us to use the same modulator/demodulator for all the subchannels and thereby reduces the complexity. Numerical results are presented for the second method. The results over a space of moderate dimensionality show substantial performance improvement with a small increase in the complexity.
\end{abstract}

\section{Introduction}

The design of a signal constellation is composed of selecting : (i) a basis for the channel space and (ii) a discrete set of points over this basis. The objective is to minimize the probability of error between the constellation points. A statistical channel is a channel with some statistical features obeying certain probability density function. The design of a signal constellation over a statistical channel involves some kind of averaging over the channel statistics. This results in a system which is on the average the best possible choice.

Some of the major problems associated with a statistical channel are as follows:

- A statistical phase shift which intervene with the orthogonality of the two phases in a QAM transmission. In general, this results in some loss in dimensionality.

- Statistical nature of the channel impulse response which results in: (i) a variable transmission gain (fading) and (ii),

This work was supported by Natural Sciences and Engineering Re search Council of Canada (NSERC). a variable intersymbol interference (ISI).

In this work, we assume that by using coherent demodulation, the first problem is solved and we mainly focus on the second problem.

In general, a channel impulse response of length greater than one results in ISI between successive transmissions. The ISI interferes with the orthogonality of the time multiplexed impulses. Our objective in dealing with the ISI in our block based signaling scheme is: (i) to preserve the orthogonality of the block dimensions though the channel (ii) to minimize the interference from the preceding blocks. In the proposed scheme, the basis is selected as the eigenvectors of the channel correlation matrix. This basis minimizes the product of the average fading attenuations along different dimensions. The interference from the preceding blocks (intra-block ISI) is modeled by an additive Gaussian noise. To reduce the effect of the intra-block ISI, an appropriate number of zeros are transmitted between successive blocks. The number of zeros is optimized to minimize the average probability of error. As by transmitting zeros the bandwidth efficiency decreases, this optimization procedure is specially useful when the bit rate is low.

By applying quadrature modulation, we obtain two dimensions with identical statistics from each baseband eigenvector. By using this modulation scheme, the channel is decomposed into a set of parallel two-dimensional subchannels with unequal fadings. A coherent M-PSK signal constellation is employed over each two dimensional subchannel. It remains to distribute the rate and the energy between the subchannels. We propose two methods to do this. In both methods, we impose the restriction that the average error probability for all the subchannels is the same. In the optimum method, the energy is distributed equally between the nonempty subchannels and the rate is distributed to have equal minimum distance to noise ratio. This method results in an unequal number of points per different two dimensional subconstellations and thereby increases the complexity of the modulation/demodulation operations. In the second method, all the nonempty two dimensional subconstellations have the same number of points and the energy is distributed to produce equal minimum distance to noise ratio.

As an example, the channel corresponding to the propagation of the electromagnetic wave in a mobile communication system is studied.

\section{System block diagram}

The block diagram of the system under consideration is shown in Fig. (1). We assume discrete time model and block based processing. The block length is equal to $N$. Two subsequent channel uses are separated by $t_{0}$ seconds. The energy per chan-

\subsection{1}


nel use is normalized to unity. The rate to be transmitted per second is equal to $R_{t}$. The $N \times N$ unitary matrix $\mathbf{M}$ is the basis at the channel input. By applying Quadrature Amplitude Modulation, we obtajn two dimensions with identical statistics from each column of M. A coherent M-PSK constellation is employed over each of these two-dimensional subspaces.

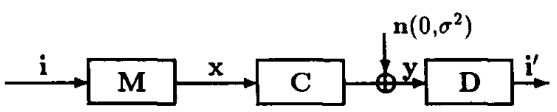

Fig. 1 System block diagram.

To reduce the effect of the intra-block ISI, $L$ zeros are transmitted between successive blocks. This results in a total of $N+L$ channel uses per each block. The idle time between two successive $N$-dimensional blocks is equal to $L t_{0}$ seconds. The rate to be transmitted per block is equal to, $R_{t} t_{0}(N+L)$.

The objective is to minimize the probability of error between the constellation points for a given $R_{t}$. Our tools are the selection of the constellation dimensions, the position of the constellation points and also the selection of the parameters $L$ and $t_{0}$.

The channel has an $N \times N$-dimensional statistical transfer matrix, $\mathbf{C}$. The $i$ 'th column of $\mathbf{C}$ is the impulse response of the channel to an impulse at time $i$. For a causal channel, $\mathbf{C}$ is a lower triangular matrix. We assume that the channel is composed of an infinite number of independent parallel subchannels. In this case, due to the law of the large numbers, the impulse response of the channel has a Gaussian distribution. In other words, the elements $C(i, j)$ of $\mathbf{C}$ are Gaussian random variables. We also assume that the elements of $\mathbf{C}$ are independent of each other and their statistics is invariant with time. In a more complex model, one can incorporate the effect of the fading memory, [1], [2], [3] or the time variance property of the channel statistics. We also assume that the expected value of $\mathbf{C}$ is equal to zero. A nonzero expected value results in a deterministic parallel subchannel. Assuming complete phase recovery (coherent demodulation), the channel transfer matrix obtains a Raleigh distribution. The additive noise is white Gaussian with zero mean and power $\sigma^{2}$.

The modulator matrix $M$ is selected such that,

$$
E\left[\mathrm{M}^{t} \mathrm{C}^{t} \mathrm{CM}\right]=\Lambda^{2} \text {, }
$$

and the demodulator matrix $\mathbf{D}$ is selected as,

$$
\mathbf{D}=\Lambda \mathbf{M}^{t}
$$

The matrix $\mathbf{R}_{\mathbf{c}}=E\left[\mathrm{C}^{t} \mathrm{C}\right]$ is denoted as the channel correlation matrix. From (1), it is seen that $M$ is equal to the eigenvectors of $\mathbf{R}_{\mathbf{c}}$ with the eigenvalue matrix $\Lambda^{2}$. The elements $\Lambda^{2}$ determine the average fading along different dimensions of $M$. It is a standard result of the matrix theory that for a fixed Trace $\left(\mathbf{R}_{\mathbf{c}}\right)$ this selection minimizes the product of the average fading attenuations along different dimensions.

As already mentioned, the intra-block ISI is modeled by an additive Gaussian noise. The assumption of Gaussianity is justified by considering that the cliannel transfer matrix has a Gaussian distribution. Using this model, the noise at the demodulator output is composed of two components, say, $\mathbf{n}$ and $\hat{\mathbf{n}}$. The first component is due to the original Gaussian noise and the second component is due to the intra-block interference. The $\mathbf{n}$ component has a Gaussian distribution with the autocorrelation $\sigma^{2} \mathbf{I}$. This is independent of the modulating matrix $\mathbf{M}$. The $\hat{\mathbf{n}}$ component has a Gaussian distribution which depends on the statistics of the source and the channel. Later, we will present an analytical expression for the power of this component in the case of a fading multipath channel and an $M$-PSK modulation.

\section{Fading multipath channel}

This channel corresponds to the propagation of the electromagnetic wave from an antenna to a receiver. We consider two kinds of reflections for the wave. The reflections occurring in the immediate neighborhood of the receiver have an additive effect. Using the law of large numbers, this results in a Gaussian density for the voltage distribution. Assuming coherent demodulation, the density function becomes Raleigh.

The reflections occurring far from the receiver has a multiplicative effect on the power attenuation. We assume that the average number of such reflections is proportional to time. This results in an exponential time decay for the energy propagation. The corresponding time constant is denoted by $\tau$.

In a more complicated model, we may assume that the power attenuation due to the far reflections has a lognormal distribution. This is based on applying the law of the large numbers to the log of the multiplicative attenuations.

In this case, the probability distribution of $C(i, j), i \geq j$, is equal to,

$P_{C(i, j)}(\alpha)=\frac{2 \alpha}{b} \exp \left(\frac{-\alpha^{2}}{b}\right), \quad b=(G / A) \exp \left[-(i-j) t_{0} / \tau\right]$

where $G$ depends on the gain of the receiver and transmitter antennas and also on the distance between the transmitter and receiver, [4], and the normalization factor $A$ which is equal to,

$$
A=\sum_{k=0}^{\infty} \exp \left(-k t_{0} / \tau\right)=1 /\left[1-\exp \left(-t_{0} / \tau\right)\right]
$$

is used to keep the total energy constant.

From (3), for $i \geq j$, we obtain,

$$
E[C(i, j)]=\frac{\sqrt{\pi(G / A)}}{2} \exp \left[-(i-j) t_{0} / 2 \tau\right],
$$

and,

$$
E\left[\{C(i, j)\}^{2}\right]=(G / A) \exp \left[-(i-j) t_{0} / \tau\right] .
$$

For a causal channel, $C$ is lower triangular and the elements of $\mathbf{R}_{\mathbf{c}}=\mathbf{C}^{\boldsymbol{t}} \mathbf{C}$ have the following form,

$$
R_{c}(i, j)=\sum_{k=\max (i, j)}^{N-1} E[C(k, i) C(k, j)] .
$$

Using (5), (6) and (7) the elements of $\mathbf{R}_{\mathbf{c}}$ are found as,

$$
R_{c}(i, i)=(G / A) \sum_{k=i}^{N-1} \exp \left[-(k-i) t_{0} / \tau\right]
$$

where $i=0, \ldots, N-1$ and,

$$
R_{c}(i, j)=(G / A) \sum_{k=\max (i, j)}^{N-1} \frac{\pi}{4} \exp \left[-(2 k-i-j) t_{0} / 2 \tau\right]
$$

\subsection{2}



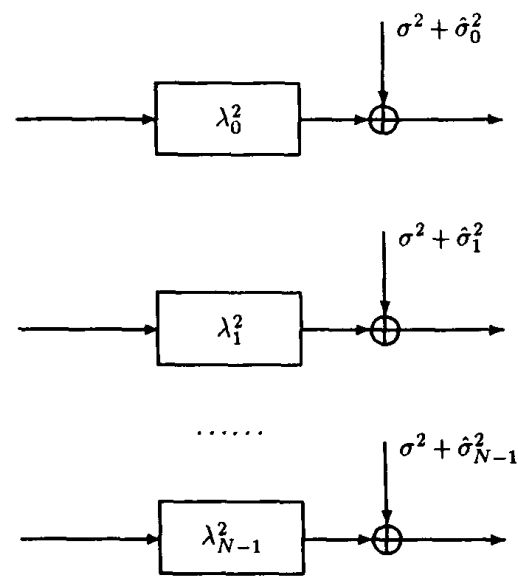

Fig. 2 The set of parallel subchannels obtained by applying QAM modulation.

where $i, j=0, \ldots, N-1, i \neq j$. Obviously, the modulator matrix $\mathbf{M}$ (the set of eigenvectors of $\mathbf{R}_{\mathbf{c}}$ ) is independent of $(G / A)$ but the fading matrix $\Lambda^{2}$ (the set of eigenvalues of $\mathbf{R}_{\mathbf{c}}$ ) has a multiplicative factor equal to $(G / A)$.

To compute the interference from the preceding blocks, define the $N \times N$ matrix $\mathrm{T}$ with the elements,

$$
T(i, j)=B \exp \left[(j-i) t_{0} / \tau\right]
$$

where,

$$
B=\frac{\exp \left[-(N+L) t_{0} / \tau\right]}{1-\exp \left[-(N+L) t_{0} / \tau\right]} .
$$

The $j$ 'th column of $\mathbf{T}$ denotes the effect of the interference from the $j$ 'th channel use within all the preceding blocks.

The correlation matrix of the data vector $i$ is equal to, $\mathbf{R}_{\mathbf{i}}=E\left[\mathbf{i i}^{t}\right]$. We assume that $\mathbf{i}$ is a white process $\left(\mathbf{R}_{\mathbf{i}}\right.$ is diagonal). The $i$ 'th diagonal element of $\mathbf{R}_{\mathbf{i}}$ denotes the power of the $i$ 'th component of $\mathbf{i}$. Let's $a_{x}(i), i=0, \ldots, N-1$, denotes the $i$ 'th diagonal element of $\mathbf{M} \mathbf{R}_{\mathbf{i}} \mathbf{M}^{t}$. This is the average power at the channel input as a function of the time index within a block. The vector $a_{x}$ is composed of the set of the elements $a_{x}(i), i=0, \ldots, N-1$. Using these notations, it is easy to show that $\mathbf{b}_{x}=\mathbf{T a} \mathbf{a}_{x}$ is equal to the power of the intra-block interference.

In summary, the interference from the previous blocks is modeled by an additive Gaussian noise with the power, $\mathrm{b}_{x}=\mathrm{Ta} \mathbf{a}_{x}$ where $a_{x}$ denotes the average power at the channel input as a function of the time index within a block. This results in a Gaussian noise of power $\hat{\sigma}_{i}^{2}$ along the $i^{\prime}$ th dimension at the demodulator output where $\hat{\sigma}_{i}^{2}$ is the $i$ 'th diagonal element of $\mathbf{D B}_{x} \mathrm{D}^{t}=\Lambda^{2} \mathbf{M B}_{x} \mathrm{M}^{t}, \mathrm{~B}_{x}$ is a diagonal matrix with the diagonal vector $\mathbf{b}_{x}$. The total power of the Gaussian noise along the $i$ 'th dimension is equal to, $n_{i}^{2}=\sigma^{2}+\hat{\sigma}_{i}^{2}$. After quadrature modulation, we obtain a set of $N$ two-dimensional subchannels with Raleigh fading, Fig. (2). The average power fading along the $i$ th subchannel is equal to the $i$ 'th diagonal element of $\Lambda^{2}$ (denoted as $\lambda_{i}^{2}$ ). The $i$ 'subchannel has an additive Gaussian noise of power $n_{i}^{2}=\sigma^{2}+\hat{\sigma}_{i}^{2}$

\section{Problem formulation}

\subsection{Signal constellation}

We have $N$ two-dimensional Raleigh fading subchannels with the average power fadings $\lambda_{0}^{2}, \ldots, \lambda_{N-1}^{2}$ and the additive Gaussian noise of power $n_{i}^{2}=\sigma^{2}+\hat{\sigma}_{i}^{2}, i=0, \ldots, N-1$. The total rate and energy are equal to, $R_{t} t_{0}(N+L)$ and $N+L$, respectively. We want to distribute the rate and the energy between the twodimensional subchannels such that the probability of error is minimized. In this case, some of the poor subchannels (with high fading and/or high additive noise) may remain empty. The number of the nonempty subconstellations is denoted by $N_{0}$. For a given $N_{0}$, the matrices $M, \Lambda^{2}$ and $R_{i}$ are of dimensionality $N \times N_{0}, N_{0} \times N_{0}$ and $N_{0} \times N_{0}$, respectively.

The proposed transmission scheme can be interpreted as a special kind of diversity. In this case, instead of transmitting the same data for several times over the dimensions orthogonal in time or frequency, we select a linear combination of the time multiplexed dimensions for a single transmission. This linear combination is the eigenvector corresponding to the largest eigenvalue. The major property of this eigenvector is that its power is concentrated near the initial part of the block. This reduces the amount of the energy propagated into the subsequent blocks and thereby reduces the intra-block interference.

The value of $L$ is optimized to minimize the average value of the error probability for a given total rate and energy. A value of $L>0$ decreases the bandwidth efficiency. In this case, the optimization procedure tries to use the available bandwidth in the best possible way. This can be also considered as an attempt to match the power spectrum of the modulator output to the channel frequency response (line coding). By increasing the bit rate, the optimum value of the idle time, $L t_{0}$, decreases. This means that the improvement caused by inserting the idle time between transmissions is higher for lower values of the bit rate. To compensate this effect for higher bit rates, one should increase the block length $N$.

We can look at this phenomenon from another point of view. Considering that the rate per block is equal $R_{t} t_{0}(N+L)$, for a given $t_{0}$, a larger $L$ results in a lower $\hat{\sigma}_{\dot{t}}^{2}$ but at the same time results in higher rate per each $N$-dimensional block. These two phenomena have opposite effects on the error probability. The selection of $L$ is based on providing the best compromise between these two effects.

Another factor is the time interval between successive channel uses, namely $t_{0}$. The selection of $t_{0}$ is based on optimizing a similar tradeoff as in the case of $L$.

The third factor is the number of the nonempty subconstellations, $N_{0}$. A lower $N_{0}$ results in a higher rate per each of the nonempty subconstellations. At the same time, a lower $N_{0}$ results in a better preformance (lower fading and lower additive noise power) for the nonempty subspaces. Again, these two phenomena have reverse effects on the error probability and the decision is based on providing the best compromise.

\subsection{Probability of error}

For an average energy $E$, the minimum distance of an M-PSK is equal to,

$$
d_{\min }^{2}=8 E \sin ^{2} \frac{\pi}{M}
$$

\subsection{3}

0290 
For an M-PSK, the decision regions are radial and consequently are insensitive to fading, [5]. In this case, assuming coherent demodulation, the probability of error is averaged over the statistics of the fading.

Assuming a Gaussian noise of power $\sigma^{2}$, the probability of error between two points of distance $d$ is upperbounded by $(1 / 2) \exp \left(-d^{2} / 8 \sigma^{2}\right),[5]$. Using this results and assuming a Raleigh fading of variance $\lambda^{2}$, the average error probability between nearest neighbors of an M-PSK is easily found as,

$$
P_{e}=\int_{0}^{\infty} \frac{a}{\lambda^{2}} \exp \left[-\left(\frac{d_{\min }^{2}}{8 \sigma^{2}}+\frac{1}{\lambda^{2}}\right) a^{2}\right] d a=\frac{1}{2}(1+\overline{S N R})^{-1},
$$

where $\overline{S N R}$ is equal to,

$$
\overline{S N R}=\frac{\lambda^{2} E}{\sigma^{2}} \sin ^{2} \frac{\pi}{M} .
$$

Using a grey code, this is approximately equal to the bit error rate, $B E R$. Similarly, the outage probability is computed as,

$$
p \equiv P[B E R \geq \epsilon]=1-(2 \epsilon)^{1 / \overline{S N R}} .
$$

We impose the restriction that the minimum distance to noise ratio and consequently the average error probability along all the subspaces is the same. The rate and the energy allocation is based on maximizing this ratio.

In the following, we propose two methods to achieve this objective.

\subsection{Optimum method for the rate and the energy distribution}

The optimum rule for the rate and energy distribution is computed from following optimization procedure :

$$
\begin{array}{ll}
\text { Maximize } & \overline{S N R}=\frac{\lambda_{i}^{2} E_{i} \sin ^{2}\left(\pi / M_{i}\right)}{n_{i}^{2}} \\
\text { Subject to: } & \sum_{\substack{N_{0}-1 \\
N_{0}-1}} E_{i}=N+L \\
& \sum_{i=0}^{N_{0}} R_{i}=(N+L) R_{t} t_{0}
\end{array} .
$$

Using the Lagrange method to solve (16), we obtain,

$$
E_{i}=\frac{N+L}{N_{0}}
$$

and,

$$
\left.R_{i}=\log _{2}\left[\frac{\pi}{\arcsin \left(\sqrt{S N R} n_{i}^{2} N_{0} / \lambda_{i}^{2}(N+L)\right.}\right)\right],
$$

where $\overline{S N} \bar{R}$ is calculated using the equation,

$$
\sum_{i=0}^{N_{0}-1} \log _{2}\left[\frac{\pi}{\arcsin \left(\sqrt{\overline{S N R} n_{i}^{2} N_{0} / \lambda_{i}^{2}(N+L)}\right)}\right]=(N+L) R_{t} t_{0} .
$$

The value of $N_{0}, L$ and $t_{0}$ are calculated to maximize the $\overline{S N R}$.

\subsection{Second method for the rate and the energy dis-} tribution

In the optimum method, the rate allocated to different two dimensional subconstellations are nonequal. This slightly increases the complexity of the modulation and demodulation operations. In the second method, all the two dimensional subconstellations have the same rate, $R_{i}=R_{0}=(N+L) R_{t} t_{0} / N_{0}, \forall i$, but the energies are different. We impose the additional constraint that $R_{0}$ is an integer. The two dimensional subconstellations are obtained by the scaling of a base M-PSK constellation with different scale factors. To provide equal minimum distance to noise ratio along all the dimensions, the energy allocation has the following form:

$$
E_{i}=\frac{(N+L) n_{i}^{2} / \lambda_{i}^{2}}{\sum_{i=0}^{N_{0}-1} n_{i}^{2} / \lambda_{i}^{2}}
$$

The unknown integers $N, L$ and $R_{0}$ are selected to maximize,

$$
\overline{S N R}=\frac{(N+L) \sin ^{2}\left(\pi / 2^{R_{0}}\right)}{\sum_{i=0}^{N_{0}-1} n_{i}^{2} / \lambda_{i}^{2}} .
$$

This is achieved by an exhaustive search.

For this problem, the matrix $\mathbf{R}_{\mathbf{i}}$ is equal to, $\left[(N+L) / N_{0}\right] \mathbf{I}$ where $\mathrm{I}$ is the $N_{0} \times N_{0}$ identity matrix.

\section{Numerical results}

In this section we present numerical results for the second method. This method employs the scaled version of the same constellation over all the subspaces. The overall complexity of the modulation/demodulation operations is low. The performance of this scheme for $R_{t}=1,8$ megabits/second and $N=1,4$ are shown in Fig. (3) and (4). The effective signal to noise ratio is equal to $G / \sigma^{2}$. The time constant of the energy propagation is selected as $\tau=60 \mathrm{~ns},[4]$. The curves corresponding to $N=1$ is used as the reference scheme. We should keep in mind that the performance of this reference scheme is also optimized over the sampling interval $\left(t_{0}\right)$ and the idle time interval between subsequent impulses $\left(L t_{0}\right)$. The increase in the complexity with respect to the reference scheme is that of two $N \times N_{0}$ linear transformations. It is seen that for the moderate value of the complex dimensionality $N=4$, the saving in energy is substantial.

Fig. (5) shows the two eigenvectors corresponding to the largest eigenvalues for $N=4$. It is seen that the power is concentrated in the initial parts of the signals. This effect reduces the intra-block interference. Table (1) shows the corresponding values of $R_{0}$ and $N_{0}$. It is seen that in most cases just one of the dimensions is nonempty $\left(N_{0}=1\right)$. From Fig. (5), the corresponding eigenvector depends approximately linearly on time. Also, in most case, $R_{0}=2$ which corresponds to a biphase signaling.

\section{References}

[1] G. L. Turin, F. D. Clapp, T. L. Johnston, S. B. Fine and D. Lavry, "A statistical model for urban multipath propagation," IEEE Trans. Veh. Technol., vol. VT-21, pp. 1-9, February 1972 . 


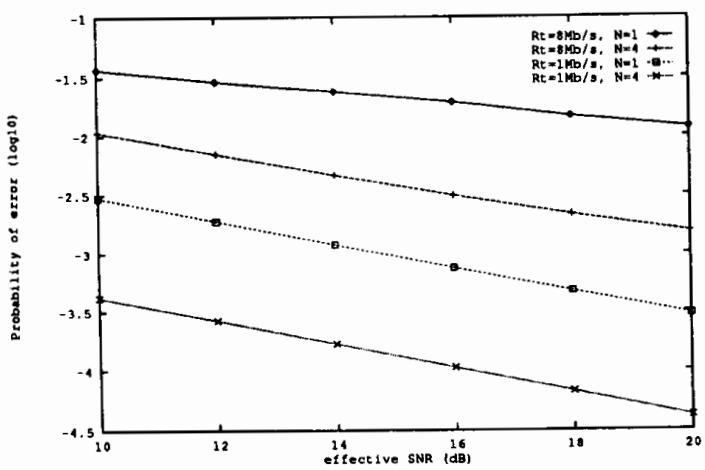

Fig. 3 Probability of error $\left(\log _{10}\right)$ as a function of the effective signal to noise ratio $\left(G / \sigma^{2}\right)$.



Fig. 4 Probability of outage $\left(\log _{10}\right)$ for $\epsilon=0.01$ as a function of the effective signal to noise ratio $\left(G / \sigma^{2}\right)$.

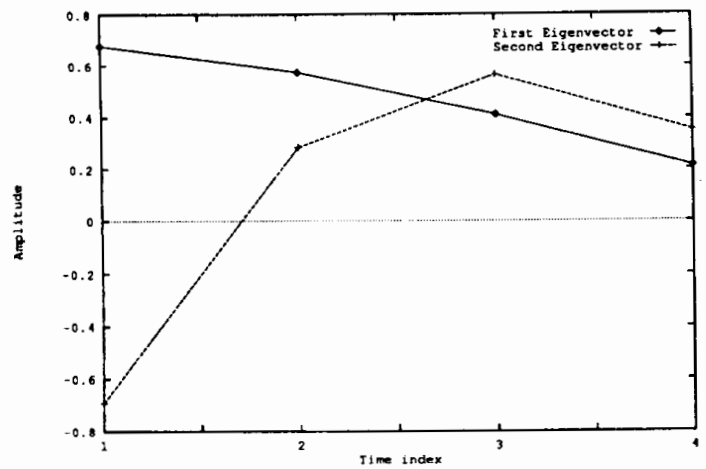

Fig. 5 The two eigenvectors corresponding to the largest eigenvalues for $N=4$.

\begin{tabular}{|c|c|c|}
\hline & $R_{t}=1 \mathrm{Mb} / \mathrm{s}$ & $R_{t}=8 \mathrm{Mb} / \mathrm{s}$ \\
\hline$N=1$ & $R_{0}=2, N_{0}=1$ & $R_{0}=2, N_{0}=1\left(G / \sigma^{2} \leq 12\right)$ \\
& & $R_{0}=3, N_{0}=1\left(G / \sigma^{2}>12\right)$ \\
\hline$N=4$ & $R_{0}=2, N_{0}=1$ & $R_{0}=2, N_{0}=2$ \\
\hline
\end{tabular}

Table 1 The values of $R_{0}$ (rate per two-dimensional subspaces) and $N_{0}$ (number of the nonempty two-dimensional subspaces).

[2] H. Suzuki, "A statistical model for urban radio propagation," IEEE Trans. Commun., vol. COM-25, pp. 673-680, July 1977 .

[3] H. Hashemi, "Simulation of the urban radio propagation channel," IEEE Trans. Veh. Technol., vol. VT-28, August 1979.

[4] A. A. M. Saleh and R. A. Valenzuela, "A statistical model for indoor multipath propagation," IEEE J. Select. Areas Commun., Vol. SAC-5, No.2, pp. 128-137, February 1987.

[5] J. M. Wozencraft and I. M. Jacobs. Principles of Communication Engineering, John Wiley \& Sons, New York, NY, 1965.

\subsection{2 .5}

